\title{
The attentional focus of expert golfers in training and competition: a naturalistic investigation
}

\author{
Marjorie Bernier \\ INSEP, French Institute of Sport and Université de Caen Basse-Normandie \\ Romain Codron \\ INSEP, French Institute of Sport and French Armed Forces \\ Emilie Thienot \\ University of Western Australia and Western Australian Institute of Sport \\ Jean F. Fournier \\ INSEP, French Institute of Sport
}

\begin{abstract}
Address correspondence to Marjorie Bernier, INSEP, Service Recherche 11, Avenue du Tremblay, 75012 Paris, France. E-mail: marjorie.bernier@insep.fr
\end{abstract}

This study explored the attentional focus of expert golfers using a naturalistic approach. Eight male professional golfers were filmed in two contexts (training and competition). Self-confrontation interviews based on the video were conducted immediately afterwards. Qualitative data analyses showed that golfers used various attentional foci. Foci were classified according to their content and their characteristics. Golfers adapted their attentional foci to the context. They used sequences of attentional foci and moved from one attentional focus to another when they prepared, executed, or evaluated their shot. Future research on attentional focus of expert athletes should study the phenomenon over time.

Attention and attentional processes have been extensively studied in sports research in an attempt to describe and explain if and how they influence performance and motor skill acquisition. Attention is defined as "the engagement in the perceptual, cognitive, and motor activities associated with performing skills" (Magill, 2003, p. 141). Among the diverse attentional processes on which research is conducted, a considerable number of recent studies have particularly dealt with how the optimal focus of attention may differ across different levels of motor expertise (e.g., Beilock, Berthental, Carr, \& McCoy, 2004; Masters, 1992; Wulf \& Prinz, 2001). Attentional focus is a concept that is poorly defined; in the present study it is considered to be the object towards which one is directing his or her attention. Several qualifying terms have been used in relation to attentional focus: internal vs. external focus (Nideffer \& Sagal, 1998; Wulf \& Prinz, 2001), broad vs. narrow focus (Nideffer \& Sagal, 1998), proximal vs. distal focus (Bell \& Hardy, 2009; McNevin et al., 2003), associative vs. dissociative focus (Morgan \& Pollack, 1977; Schomer, 1986), and endogenous (voluntary) vs. exogenous (non-voluntary) focus (Jonides, 1981; Posner, 1980). These different conceptualizations highlight the complexity involved in the concept of focus.

In a series of studies (for a review, see Wulf \& Prinz, 2001), Wulf and colleagues demonstrated that focusing on the outcomes of the movement (external focus) was associated with greater success than focusing on the movement execution itself (internal focus). McNevin et al. (2003) explained the superiority of external over internal focus for performance and learning through the constrained action hypothesis. According to this 
hypothesis, the adoption of an internal focus causes the actor to actively intervene in the posture and/or the movement. It is thought that active intervention in automatized processes inadvertently disrupts the coordination of a number of relatively automatic processes that would typically control the movement (Wulf, 2007).Attempting to consciously control movements may therefore degrade, rather than enhance, the movement outcome. In contrast, the advantage of an external focus of attention may be to allow unconscious or automatic processing to control the movements required to achieve the expected effect (Wulf, 2007).More recently, Wulf and Su (2007) reported the same effect in novice and expert golfers performing a chipping task. They showed that instructions inducing an external focus (in their study, on the motion of the club) resulted in significantly higher accuracy scores, irrespective of the skill level of the participant, when compared to either an internal focus (on the motion of the participant's arm), or in a condition providing no instructions for attentional focus (control condition).

In contrast to the results of Wulf and $\mathrm{Su}$ (2007), numerous studies have reported that optimal attentional focus may differ according to the skill level of the participant (Beilock et al., 2004; Beilock, Carr, Mac Mahon, \& Starkes, 2002; Ford, Hodges, \&Williams, 2005; Singer, 2002). For example, Beilock et al. (2002) examined how an internal and external focus of attention influenced the dribbling skills of novice and skilled footballers. Whereas the novice athletes performed best when focusing on the movement execution process (internal focus), internal focus was counterproductive for the experts, who had automatized their skills and performed best with an external focus of attention. Perkins-Ceccato et al. (2003) replicated these results in golf. They found that highly skilled golfers performed more effectively with instructions guiding towards external focus (i.e., to focus on hitting the ball as close to a target as possible), whereas less skilled golfers benefited more from instructions encouraging an internal focus (i.e., to focus on the movement form). According to these results, the effectiveness of the attentional focus will differ according to the level of skill acquisition.

Bell and Hardy (2009) have recently extended this research theme by comparing the effectiveness of different locations of focus in a sample of skilled golfers experiencing different levels of anxiety. They distinguished three foci: internal focus (i.e., the motion of the arm during the swing), proximal external focus (i.e., the position of the clubface throughout the swing), and distal external focus (i.e., the flight - and in particular the direction - of the ball).

Bell and Hardy found that skilled golfers who used a distal external focus performed most accurately, whereas golfers who used an internal focus performed least accurately, regardless of the anxiety condition.

Anxiety is an important issue to address when studying the attentional focus of expert athletes, particularly considering the apparent link between anxiety and attentional processes in the phenomenon of choking. Choking happens in high-pressure situations and is linked to a considerable desire to perform well (Hardy, Mullen, \& Jones, 1996). A number of studies directly addressing choking have enhanced our understanding of how attentional focus influences expert performance. Two particular theories have sought to explain choking using different interpretations of how pressure impacts performance: distraction theories (Eysenck\& Calvo, 1992; Wine, 1971), and explicit monitoring theories (e.g., Baumeister, 1984; Beilock \& Carr, 2001; Masters, 1992). Distraction theories postulate that anxiety demands a large allocation of attentional resources. Pressure situations inducing anxiety therefore prevent athletes from solely focusing on the task at hand. Explicit monitoring theories on the other hand suggest that pressure situations that induce anxiety will result in changes in attentional focus. By seeking to function at one's best, athletes under anxiety turn their attention inwards to the specific processes of performance. As a result, they perform more explicit monitoring 
than they would do in a non-pressure situation. Focusing on skill execution in a step-by-step manner degrades performance for a well-learned automatized skill (Beilock \& Carr, 2001).

Similar to these explanations of choking is Masters' (1992) theory of reinvestment, suggesting that performers attempt to consciously control their actions in pressure situations, reinvesting their attention in well-learned processes of skill execution. This inappropriate focus leads to the disruption of expert performance, and as a result can decrease performance and create opportunities for error. Beilock et al. (2002), on the other hand, have indicated that attentional focus on skill processes may not always be detrimental to expert performance. Attention to skill execution can be beneficial to alter or change performance processes. Expert athletes may need to slow down and dechunk previous execution processes in an attempt to modify them and produce performance benefits (Beilock et al., 2002). Gucciardi and Dimmock (2008) also showed that a focus on skill processes may be useful for expert golfers. For example, a holistic cue word or a focus on the smooth or the rhythm could be effective on performance.

In summary, literature on attentional focus in expert performance can be considered somewhat consistent on some issues and inconsistent for others. It is generally agreed that an internal focus of attention is detrimental to expert performance. Focusing on movement execution and step-by-step processing interferes with what should be automatic skills that characterize expert performance. The adoption of this type of focus is observed in situations that induce anxiety - such as in high-level competition - and often is seen to manifest itself as choking.

Other results are more equivocal, in particular those related to the location of the external focus. The inconsistencies in the literature may be due to a lack of agreement on the underlying concepts. Researchers have used different terms (e.g., external vs. internal, proximal vs. distal, endogenous vs. exogenous, associative vs. dissociative) without any consensus on their exact definition. Bell and Hardy (2009) illustrated this problem with their concept of a distal external focus. They claimed that studies that have tested the effectiveness of attentional focus were based on different operational definitions of the distal external focus. These different definitions may help to explain the contradictory results that have been reported. These distinctions between the various types of attentional focus were created to test their respective effectiveness on performance (e.g., Bell \& Hardy, 2009; Wulf \& Prinz, 2001). However, it is unclear if the use of these constructs is relevant for describing attentional foci naturally experienced by athletes. Thus, it became necessary to investigate the nature of attentional foci that emerged in natural performance situations.

To best evaluate attentional focus, studies have employed experimental designs that differ from natural situations encountered by expert athletes. In a literature review, Wulf and Shea (2002) questioned the generalizability of results reported in studies using artificial laboratory tasks. They outlined the need for more complex and ecologically valid tasks in research designs to gain further insights into the execution of motor skills processes. More recently, Oudejans, Kuijpers, Kooijman and Bakker (2010) highlighted that attentional focus must be examined in natural sport settings that are characterized by pressure conditions.

Moreover, few studies have dealt with attentional focus in expert sport. As recommended byWilliams and Ericsson (2005), the present study was designed to take into account the complex mechanisms that mediate truly expert performance in dynamic and uncertain conditions, with specific physiological and emotional demands. In a naturalistic approach, the goal was to examine attentional focus in natural settings experienced by expert golfers. Contrary to previous research in the field, we did not set out to evaluate the effectiveness of different kinds of attentional focus on performance, but rather to employ a qualitative method to explore and to characterize more precisely the attentional focus of expert golfers in natural situations of training and competition. 


\section{METHOD}

\section{Participants}

Eight male professional French golfers voluntarily participated in the study. The eight players took part in a research study for the first time, and none were clients of any of the researchers. Players were contacted during a coach education class. They were informed that their participation would advance knowledge in golf performance, and that they would be informed about the results of the study during classes given to coaches by the National Golf Governing Body. Out of the nine players in the class, eight responded that they would volunteer to take part in the study. Participants were $24-37$ years old $(M=30.87, S D=4.42)$ and had practiced golf for $11-23$ years $(M=19.00, S D=4.41)$. At time of the study, they trained 18 to $30 \mathrm{hr}$ a week $(M=26.00, S D=4.97)$. Various other characteristics allowed for appraising their level of expertise in golf. First, they were all good amateur players before turning professional.

Each participant was at least selected once to play on the national team for an international tournament. Second, all participants played on various professional tours. One participant was a regular European Tour player, a second had partial access to the European Tour while three were Challenge Tour players and the last two played on the Alps Tour. Their participation in these professional competitions indicates a high level of performance in competitive golf to have access or maintain access at a professional tour. All players signed an informed-consent form and were given pseudonyms to provide anonymity.

\section{Protocol}

The study was carried out in two phases: (a) during a training session, and (b) during competition.

\section{Training session}

All participants were first filmed during a training session. This phase of the study took place during the winter (non-competitive) season. Participants had not competed for one month, and the next competitive season started one month following this training session.

Each participant was filmed for a duration of $1 \mathrm{hr}$ during his individual training session. Prior to training, participants were informed that the goal of the study was to better understand the attentional processes of expert golfers. Participants were instructed to train as usual and to do what they had planned to do in that session. Golfer 1 spent his training session on various chipping situations around the green. He had to try and place three balls less than $1 \mathrm{~m}$ away from the hole. Golfer 2 used first a mirror to work on his putting for short putts. He then tried to place five balls in a row not further away than $50 \mathrm{~cm}$ from the hole. Golfers 3 and 5 worked on their swing at the driving range with irons using various educational tools. Golfer 4 worked on ball trajectories at the driving range with various clubs. Golfer 6's training sequence was divided into some work at the driving range and a second session on the putting green. The first part of the session aimed to enhance posture (straighter) with all the clubs. On the putting green he went through various slope reading exercises and speed exercises before ending with a pressure exercise in which he had to hole a given number of balls in pre-defined holes. Golfer 7 produced all the ball trajectories he could perform at the driving range, before working on putt rhythm with a tool attached to his club. Golfer 8 worked on his short game. The first exercise sought to enhance chipping by playing the same zone on the green three times, using one hand. 
Then, he worked on imagery in putting by alternating putts with eyes open and eyes closed, with and without pressure. All were informed that they would be filmed. Self-confrontation interviews (von Cranach \& Harré, 1982), based on video footage, were conducted less than 2 $\mathrm{hr}$ following training.

\section{Competition}

The second phase of the study took place 5 months later, during the competitive season. Six of the 8 participants volunteered to take part in the second phase. Each of the 6 golfers was videotaped at a professional competition (National Tour) during a complete round (i.e. 18 holes of play) that lasted approximately $4 \mathrm{hr}$. To prevent distraction for participants prior to competition, information about the study (the goal of the study and the video recording conditions) was provided for participants one week prior to competition. No other instructions were given before the round. Self-confrontation interviews were conducted based on the video, and took place no longer than $2 \mathrm{hr}$ after the completion of the round.

\section{Data collection}

Qualitative data were collected from self-confrontation interviews to recall attentional focus. The interview was based on techniques of stimulated recall (von Cranach \& Harr'e, 1982; Trudel, Haughian, \& Gilbert, 1996), consisting of showing the participants his own activity in particular situations as soon as possible after the event. Although retrospective recall procedures may be perceived to result in incomplete data collection due to memory loss and an inability to access cognitive processes that do not reach consciousness (Wilson, 1994), there is considerable evidence in the sport psychology literature to support the use of these procedures in the recall of cognitions or emotions (e.g., Hanton, Mellalieu, \& Hall, 2004; Tenenbaum, Lloyd, Pretty, \& Hanin, 2002). Moreover, we could not question the participants about their thoughts during play because it would have been distracting. Thus, the selfconfrontation interview with video appeared to be a useful compromise, as it served as a stimulus for recall. In addition, this study focused on conscious processes, with no requirement to reveal unconscious processing.

Self-confrontation interviews were based on the video images of the participant during the training session or competition. As the participant viewed the videotape with the researcher, he was invited to recall and describe what thoughts he was personally experiencing during the training session or during competition. We chose to select sequences to watch with the participants because it was impossible to watch all the footage $(1 \mathrm{hr}$ per training session and about $4 \mathrm{hr} 30 \mathrm{~min}$ for the competition). The choice of the sequences watched respected the following criteria for all the participants. First, the participant and the researcher watched the first situations on the video recording, which are the first training exercise and the first three holes of the competition. Once attentional foci of these situations were described, the researcher asked the participant to indicate specific situations that he considered relevant to analyze. Thus, the researcher and the participant chose together and watched some significant situations. These situations were related, for example, to specific exercises during the training session or to some great shots or poor shots during the competition. Each sequence included an action (e.g., a training drill or a shot), the preparation phase (e.g., the routine), and the step following this action (e.g., putting the club in the bag or walking to the ball). In the training phase, the number of sequences watched during the interview with each participant was between 10 and 14. During the competition phase, it was between 15 and 17. For each of these sequences, the participant was asked to freely indicate and describe any thoughts related to attentional focus that he remembered. The participant or the researcher would pause the 
reading of the tape to leave time for describing in depth the thought occurring at this specific point in time. The function rewind was also used to make sure that all the attentional foci that occurred during the given situation were mentioned by the participant.

The researcher urged the participants to describe the internal events and the thoughts experienced simply, and avoided requests for generalization and self-analysis. Probe questions were designed to collect the nature (object) of the attentional foci, that is, all events that were consciously accessible in the given situation (e.g., participants would be asked "What are you thinking about at this moment?"). These questions were asked to elaborate on the participants' answers, and to gain a better understanding of their attentional focus. Prompts concerned only the golfer's action or attentional focus during the training session or competition, with no justification for the focus sought or required. The interviewers attempted to collect the verbal expressions of the participants' thoughts, rather than asking them to explain their solution for the task or to provide a summary of the general strategy adopted. Interviews were videotaped by a camera placed behind the participant's chair so that the video images shown to participants were within the field of view of the camera. All interviews were conducted in French by the first and the second author because it was the native language of all the participants and researchers. Interviews lasted between 1 to $1.5 \mathrm{hr}$.

\section{Data analysis}

Interviews were transcribed verbatim and subjected to inductive content analysis. In the first stage, two researchers independently identified units of meaning (MUs), corresponding to attentional foci reported by the participant. They processed an inductive coding of 688 identified MUs. The number of MUs collected in each interview with a golfer varied from 31 MUs (Golfer 8) to 62 MUs (Golfer 2) in the training phase and from 29 MUs (Golfer 7) to 84 MUs (Golfer 2) in the competition phase. In the second stage, all MUs were compared and grouped together (according to thematic similarities) into emergent sub-categories (Lincoln \& Guba, 1985; Patton, 2002). The sub-categories were then further grouped into increasingly more complex categories according to common features. During the third stage, an examination of the sequences of attentional foci was conducted. It consisted in observing the succession of attentional foci.

\section{Validity and trustworthiness}

In agreement with Sparkes and Smith (2009), we identified and tried to respect criteria that can be considered as trustworthy in the specific context of this study. First, the selection of a sample of elite golfers representative of the population of expert golfers enhanced the transferability of the findings of this study. Second, the four authors were sport psychology researchers.

They had extensive experience in traditional qualitative research and self-confrontation interviewing techniques. They had conducted at least four studies in sport psychology based on qualitative methods and had therefore developed interviewing and analyzing skills. The first, second, and fourth author had acquired robust knowledge about golf expertise through their experience as sport psychology consultants in golf. In their consultation practice, they had noticed that the application of the results of experimental studies on attentional focus was difficult. This is one of the reasons that led them to work on this theme with a naturalistic approach, and to design the present study. Their involvement established credibility as a result of prolonged commitment to, and long-term observation of, the sport and its players.

The first and second author conducted the interviews and coded the transcripts. The two analysts completed the inductive coding of the transcripts independently. Then five debriefing 
meetings between the two analysts and the fourth author (for peer debriefing) were held to elicit the various and divergent codings. Comparison and discussion of the codings and categorizations occurred until a consensus was reached. The third author, a psychology researcher belonging to a different institution from the first two authors, performed an inquiry audit and an independent coding. She had no experience of research in attentional focus or in the sport of golf.

She was trained in qualitative methods and unaffiliated with this study. This independent audit provided a means of cross-validation and ensured that the authors' categorizations were valid. A sample of the data $(20 \%$ or $138 \mathrm{MUs})$ was extracted randomly from each interview and given for coding to the third researcher. She coded the sample of units with the grid of analysis conceived by the three other researchers during the coding of all MUs. Inter-coder agreements between the first coding and the independent coding were computed with a Kappa index $(k$; Cohen, 1960). This indicator appraises the normalized proportion of inter-coder agreement in excess of what would be expected on the basis of chance or random assignments. MacKappa software (Watkins, 2002) was used to compute general coefficients and to test the statistical significance of agreement among codings based on Fleiss computational formulae (1981). The various Kappas (see Table 1) indicate a good rate of agreement among coders. These results strengthen the validity of the coding process. Member checking was addressed by sending the research report to all of the participants and by asking them to comment on any part of the report. None of the participants answered, but two participants seen during tournaments mentioned that the results expressed clearly what they experienced during training and competition.

\section{RESULTS}

Table 1 presents the emergent categories and sub-categories that were used to classify each of the 688 MUs (688 attentional foci), corresponding to the different attentional foci described by the participants. The number of MUs in each category and percentages are indicated to provide elementary and descriptive information about the coded qualitative data. In the results section, emergent categories and sub-categories are illustrated using qualitative data.

Categorization revealed that each element of attentional focus could be defined by content (i.e., the object of the golfer's attention), and by different characteristics (i.e., how the golfer pays attention to this object). Following a consideration of these categories, other results are reported addressing (a) the situations (in training and in competition) in which attentional focus emerged, and (b) the sequences of attentional foci.

\section{Categories based on content}

Attentional foci were organized according to their content, that is to say, the object of the attention of the golfer. Four categories of content were identified through inductive coding: process, result, psychological state, and environment (see Table 1 for categorization of MUs). The process category (294 MUs, $42.73 \%$ of data on foci content) involves a focus on the swing or on the movement. In particular this category involves attentional focus on technical aspects, procedural rules, body positions, and club motion. Examples include the following: "I pay attention to the position of my right hand" (Golfer 4) or "I focus on the rhythm of my swing" (Golfer 2).

The second category of content is the result (272 MUs, $39.53 \%$ of data on foci content), which includes all attentional foci addressing the outcomes of the movement or of the process, for example, "I focus on where the ball will land" (Golfer 7) or "I visualize the trajectory of the ball" (Golfer 2). Two particular sub-categories were identified in the result category 
corresponding to effects more or less close to the process: (a) outcomes related to the ball (e.g., trajectory or roll of the ball, landing point, the ball falling in the hole), and (b) outcomes relevant to the score (e.g., "Here, I thought that if I made one more birdie I would certainly reach the cut," Golfer 1).

The third content category clustered all attentional foci related to psychological state ( 74 MUs, $10.75 \%$ of data on foci content). These attentional foci corresponded to emotional and motivational states. For example, golfers focused on their anxiety level, or on their confidence or other states: "At this time, I suddenly felt pressured because I couldn't fail" (Golfer 5) or "Before this shot, I was very confident, I knew that the ball would get to the hole" (Golfer 7). The fourth and final category of content that emerged from the qualitative data was the environment category (48 MUs, $6.98 \%$ of data on foci content). Sometimes golfers allocated their attention to surrounding elements. These environmental aspects may or may not have been related to the specific golf task. Therefore two sub-categories of environment foci arose: (a) Task-related environmental foci, providing information that contributed to the decisionmaking process and to a strategic or tactical choice. For example, "I check a complete list of information before choosing my shot: the distance, the wind, the lie of my ball" (Golfer 2). (b) Environment elements that were not directly related to the golf task, and could be considered a distraction, for example, "A bird landed in my visual field when I addressed the ball" (Golfer 7) or "Helicopter noise caught my attention" (Golfer 3).

\section{Categories based on characteristics}

Rather than categorization of attentional foci based on content, qualitative data analysis allowed alternate means of categorization based on important characteristics of attentional foci. These characteristics consist in how the attentional focus arises, or how it was used by the golfer. In this categorization, attentional focus was classified to each of three different characteristics that emerged from the inductive coding: sense, reality, and deliberateness.

The characteristic of sense was further subcategorized into kinesthetic (e.g., "I focus on the sensation of release in my hands," Golfer 6), visual (e.g., "I look at the position of my right hand on the shaft," Golfer 1), or auditory (e.g., "I focus on the impact noise," Golfer 5) sensations (see Table 1 for categorization of MUs).

The second classification was based on the characteristic of reality. Real and imagined foci were distinguished (see Table 1 for categorization of MUs). Real foci were concrete and available objects that participants could attend to, for example, "Before the shot, I stand behind the ball, I focus on the slopes, I analyze their inclination degrees and their directions" (Golfer 1). In contrast, imagined foci are objects created by mental imagery: "I visualize the complete roll of the ball. I see it going in reverse from the hole to the tee and vice versa" (Golfer 2).

The third characteristic addressed the deliberateness of attention (see Table 1 for categorization of MUs). A deliberate focus is intentional and controlled by the golfer. The following sentence illustrated this kind of focus: "To reach this target, I must focus on the rhythm" (Golfer 6). In this case, the golfer deliberately paid attention to something (i.e., the rhythm), choosing the focus that he felt was most appropriate. In contrast, a spontaneous (non-deliberate) focus is non-intentional or involuntary, with thoughts and attentional focus arising beyond conscious control, for example,

Here in this exercise, my goal is to hole so many balls in a row. So I focus on the result, not on the technical elements from earlier on. But still the technical elements are there [in my mind], even if I did not want to think about them (Golfer 4). 
As Golfer 4 planned not to focus on technical elements, he did not seem able to control all the foci that arose. Other uncontrolled thoughts are mentioned by Golfer 2:

And then, I was one ball away from completing the exercise. I tried to keep my usual preparation, but I thought. . watch out. . .even the best golfers can't hole three metre putts one hundred percent of the time. I could not help thinking of the result (Golfer 2).

\section{Categories of focus in training and in competition}

The various categories and sub-categories of attentional focus emerged differently in each situation in which they were observed (in training and in competition). Considering the percentages, each category of foci represents different parts of all MUs, depending on the situation (see Table 2). Data highlight that process foci represent $60.33 \%$ of all MUs identified in training, whereas they represent only $29.20 \%$ of all MUs in competition. In contrast, result foci represent $31.67 \%$ of all MUs in training, and $45.73 \%$ in competition. Environment and psychological state foci arose most commonly in competition. They represented a small portion of all the MUs in training (2.33\% and 5.67\% respectively). Visual foci represented $57.60 \%$ of all MUs in training, whereas they represented $73.03 \%$ of MUs in competition. Conversely, kinesthetic foci represented $40.99 \%$ of the MUs in training, and $26.67 \%$ in competition.

\section{Sequences of attentional foci}

The qualitative analysis of attention revealed sequences that appeared to occur in the reports of participants, even over a short period of time. For example, when Golfer 5 reported the attentional foci that he used in his pre-performance routine for a drive at the 9th tee, a series of attentional foci emerged:

So here we are at the ninth tee. I start to take in information: the distance, the wind. I place my tee, and just after that I focus on the target I have chosen. Here it was the tree shadow on the left. I take my practice swings and at the same time I focus on letting go, and I try to feel the rhythm well. There. And here I walk to the ball. I visualize the trajectory, only the ball at the start. I address the ball and I look at my target one last time ... and bang, I shot.

Golfer 5 shifted his attentional focus across a series of foci: (a) "information" (visual real deliberate environment foci), (b) "the target I have chosen" (visual real deliberate result focus), (c) "the release and rhythm feelings" (kinesthetic real deliberate process foci), (d) "the ball start trajectory" (visual imagined deliberate result focus), and (e) "the target" (visual real deliberate result focus). Foci with different kinds of content and characteristics arise in succession to allow Golfer 5 to prepare his shot.

Many other sequences of attentional foci were observed in the pre-performance routines of participants. Sequences were also identified for other situations, for example, when golfers evaluated their shot, or when they were disturbed by environmental distractions and/or their thoughts (e.g., negative emotions or feelings). For example, Golfer 2 focused successively on foci with different content and characteristics between two trials in a training exercise:

I totally missed this shot. I got bad sensations upon contact with the ball [kinesthetic real spontaneous process focus] and the ball trajectory was too low [visual real deliberate result focus]. So I tried to call to mind the sensations that I should feel when making contact with the ball [kinesthetic imagined deliberate process focus] and to focus on the ball-height by imagining the whole trajectory [visual imagined deliberate result focus]. 


\section{DISCUSSION}

\section{Content and characteristics of attentional focus}

The goal of this qualitative study was to examine and describe the attentional focus of expert golfers in training and competition natural settings. Exploring and describing attentional foci of expert golfers provided results suggesting new distinctions and classifications of attentional foci. Classifying attentional focus according to content (process, result, environment, psychological state) and characteristics (sense, reality, deliberateness) supplements existing distinctions highlighted in the literature. Previous distinctions in the literature compared two or three kind of foci. For example, the differentiation of internal and external focus (Nideffer \& Sagal, 1998; Wulf \& Prinz, 2001) provided little specificity for the object of the athlete's attention. In the present exploratory research, it appeared that attentional foci of expert golfers were more diverse and more specific than the kinds of foci previously examined in the literature. Several categories of focus emerged and could constitute a framework to classify attentional focus. First, results demonstrated that expert golfers focused on specific elements that constitute specific categories of content. Until now, this notion of content of attentional focus has never been highlighted; hence, the different categories of content on which athletes focus have never been identified. Thus, this distinction based on content can enhance our knowledge and understanding of the notion of attentional focus. Moreover, it could help athletes and coaches to identify the specific elements that athletes focus on more precisely. Further work is necessary to examine whether this distinction based on content is relevant to the examination of attentional foci in other sports. For example, the "effects on the ball" subcategory can be used solely in ball sports. Preliminary evidence exists to suggest that content of attentional focus is dependent upon the characteristics and requirements of the sport. It is therefore necessary to replicate this kind of research in order to improve our knowledge of content of focus depending on the specificity of sports. The emergence of psychological state as a category of attentional focus is relevant because it demonstrates the impact of these internal events (emotions, perceptions of motivation or confidence) on the attentional process. Indeed, when golfers attended (either deliberately or not) to their anxiety state or confidence state, it constituted a particular category of focus. The beneficial or detrimental effect of psychological state foci on performance emerges as a specific issue worthy of further research, and one that could be considered using explicative theories of choking under pressure (e.g., Beilock \& Carr, 2001; Eysenck \& Calvo, 1992; Masters, 1992).

Second, the observed characteristics of attentional focus also provide new perspectives into the attentional focus of expert athletes. It is important to consider these outcomes in relation to the existing knowledge of different fields of sport psychology. For example, the sense characteristic allows an important link to be made between the notion of attentional focus and the perceptual and information processing literature (Magill, 2003). Complementing a traditional cognitive approach, the present results show that expert golfers in natural situations use different information sourced from visual, kinesthetic and auditory attentional foci. The sense characteristic could be helpful when considering the perceptual mechanisms in an applied setting.

The reality characteristic is particularly relevant to research in mental imagery. Expert golfers were shown to use mental imagery to focus on specific objects $(20.06 \%$ of the reported attentional foci were imagined). The link between attentional focus and mental imagery has been previously highlighted (e.g., Caliari, 2008; Fournier, Deremaux, \& Bernier, 2008; Murphy, Nordin, \& Cumming, 2008). The functional equivalence hypothesis postulates that the perceptual processes involved with imagery share some neural pathways and mechanisms with the preparation and production of motor movements (Jeannerod, 2001). This theory has 
an important implication in the applied setting, because imagery is considered by some researchers (e.g., Calmels, Berthoumieux, \& d'Arripe-Longueville, 2004; Loze, Holmes, Collins, \& Bellamy, 1998) as a means to focus attention on relevant and appropriate cues. In this study, it appears that golfers used imagery as a strategy to focus on different elements of their game, as evidenced by reality emerging as a characteristic of attentional focus.

The characteristic of deliberateness is one that has received very little attention in sport psychology. Boutcher (2008) specified that according to the attentional selectivity perspective (Posner \& Boies, 1971), the selection of information can be deliberate or involuntary depending on whether selection originates in the organism or is due to the stimuli itself. In visual and spatial attention, a distinction has been made between endogenous (deliberate) attentional focus, which is orienting, determined, and controlled by the subject's will, and exogenous (reflexive or spontaneous) orienting, which is usually out of the subject's control and is brought about by the abrupt onset of a peripheral stimulus. In sport psychology, this characteristic of attentional focus has rarely been considered, one exception being Nordin and Cumming (2005), who showed that professional dancers experienced both spontaneous and deliberate mental images. However, in the results of the present study, this characteristic emerged as important, with $11.92 \%$ of foci being spontaneous. It may be interesting to establish the influence of involuntary foci on performance, and furthermore to study the strategies used by athletes to manage them. Indeed, some intrusive thoughts might be perceived as debilitative by the athletes and then potentially distracting or disturbing (e.g., imagining failure, worries about the cut).

They may lead to choking because they require attentional resources (distraction theories), or because they induce doubts that may lead athletes to try to change their attentional strategies in an attempt to consciously control their automatized movements (explicit monitoring theories).

In summary, the classification that emerged from the analysis of data is related to many relevant themes in cognitive psychology (e.g., the perceptual process, mental imagery, deliberateness in visual attention) that have not been explicitly addressed in previous studies considering attentional focus. These associations between the various notions of attentional focus and other themes in cognitive psychology are worthy of further research in sport psychology.

These new classifications can also be applied to better understand the attentional focus of athletes.

\section{Variation and sequences of attentional foci: a perspective over time}

The results demonstrated that attentional foci varied according to the situation in which the golfers were embedded; they experienced different foci according to whether they were in training or competition. For example, reports revealed that content concerned with result occurred mostly in competition, whereas process content was most frequent during training. The two situations in which attentional focus was studied were very specific according to their naturalistic dimension. It has been suggested that, given the complexity and variability of each specific performance situation, an examination of focus over time may be an appropriate approach for studying the attentional foci of expert athletes. Practical implications are considered and deserve further examination: considering that the coaches' instructions play an important role in the attentional foci used by the athlete, it would be interesting to examine how coaches adapt their instructions according to the specific context of performance.

The dynamic aspect of attentional focus was also observed through emergent series of foci. With these series of attentional foci, golfers shift over a short period of time from one attentional focus to another when they prepare, execute or evaluate their shot. By highlighting 
that the series of foci refers to a complex attentional process, these results make available an important advance compared to previous studies. Until now, experimental research has studied the effectiveness of a unique focus, without identifying when participants used this type of focus (i.e., before, during, or after the action). In these sequences of attentional foci, one can observe that golfers successively focused on different kinds of content, and with different characteristics to prepare for, execute or evaluate the same action. These results are linked to the concept of a pre-shot routine defined as "a set pattern of thoughts, actions, and images consistently carried out before performance of the skill" (Crews \& Boutcher, 1986, p. 62).

More specifically, these series of foci highlight the attentional component of the routine. The pre-shot routine can be considered as a strategy to focus on an appropriate series of varied content with different characteristics. In the current study, all the players used a pre-shot routine during competition. During the training situation, all the players also clearly used a pre-shot routine during specific exercises, or when their training session consisted in playing on the course.

Finally, the dynamic perspective emerged as a central consideration in the notion of attentional focus. Golfers experienced series (or sequences) of foci that were adapted to the specific conditions participants face. The static and dichotomous approach used in the literature (i.e., experimental studies comparing the efficiency of a given type of focus versus another one) was never sufficient to examine the dynamic and adaptative aspect. Hutchinson and Tenenbaum (2007) showed that attentional foci of athletes during long duration tasks varied from a dissociative mode (i.e., focused on object not linked to the task, such as distraction) to an associative one (i.e., focused on effort perception) along with task intensity. When we processed the member checking, two participants insisted that variations and adaptations of attentional focus were crucial in their practice. They considered that they should work on their attentional skills and take into greater consideration this dynamic aspect of attention. Singer (2002) addressed this perspective by emphasizing the self-organizational capabilities of skilled athletes through the interaction of the self and the environment. In terms of applications, this highlights the need to develop skills of self-regulation in order to endow athletes with the capacity to adapt and manage their attentional focus in the specific situations they encounter.

\section{Strengths and limitations of the study}

Although this study has made important contributions in furthering the understanding of attentional focus, there are a number of limitations that must be addressed. The low sample size is a particular limitation that confines the generalizability of the findings, an issue that is often unavoidable in the examination of expert athletes. Moreover, it was unfortunate that the number of participants decreased from 8 in the first phase (training session) to 6 in the second phase (competition). We could not force the volunteer golfers of the first phase to participate in the second phase because of the important stakes of this professional competition, in particular financial stakes. The two golfers who did not participate in the second phase were afraid of being disturbed by the study conditions during their rounds. The 6 participants who volunteered for the second phase confirmed during the interview that they were not at all disturbed by the study conditions.

Despite these limitations, the present study presented important methodological strengths. All participants play at an expert level, ensuring that truly skilled athletes were examined. The protocol also allowed expert performers to be studied in a natural setting. This supplemented previous results in an innovative manner. As indicated by Abernethy, Thomas, and Thomas (1993), the novel nature of simplistic or contrived laboratory tasks is likely to alter expert 
processing (e.g., information processing, problem-solving). The naturalistic dimension of this study takes into account the complexity and variability of situations encountered by expert athletes, providing a more realistic evaluation of what takes place in a natural setting.

\section{Perspectives}

A number of issues raised but not explicitly explored in this study deserve further consideration. First, research on other cognitive processes (e.g., observational learning: Cumming, Clark, Ste-Marie, McCullagh, \& Hall, 2005; Hars \& Calmels, 2007; imagery: Fournier et al., 2008; Murphy et al., 2008) have revealed functions to be an important concept. In imagery, Fournier et al. (2008) showed that expert golfers used specific content and characteristics in their imagery according to the function of the mental images (e.g., to enhance motivation, or to correct one's movement). It can be hypothesized that this functional dimension could explain the variation of attentional foci in different situations. Attentional foci could be used for different functions depending on the situation. Additionally, a second relevant issue deals with athletes' interpretation of the different kinds of attentional foci. It can be suggested that athletes perceive their attentional focus as debilitative or facilitative; that is to say that they may be perceived to have negative or positive effects on performance. This interpretation could induce different strategies to accept or manage their foci. A variety of complementary approaches could be helpful to investigate these research perspectives. Some specific tools could be developed using new technologies (e.g., video, virtual reality, eye-movement measures) to better understand the dynamic process that regulates attentional focus.

\section{REFERENCES}

Abernethy, B., Thomas, K. T., \& Thomas, J. T. (1993). Strategies for improving understanding of motor expertise (or mistakes we have made and things we have learned!!). In J. L. Starkes \& F. Allard (Eds.), Cognitive issues in motor expertise (pp. 317-356). Amsterdam: Elsevier Science Publishers.

Baumeister, R. F. (1984). Choking under pressure: Self-consciousness and paradoxical effects of incentives on skillful performance. Journal of Personality and Social Psychology, 46, 610-620.

Beilock, S. L., Berthental, B. I., Carr, T. H., \& McCoy, A. H. (2004). Haste does not always make waste: Expertise, direction of attention, and speed versus accuracy in performing sensorimotor skills. Psychonomic Bulletin and Review, 11, 373-379.

Beilock, S. L., \& Carr, T. H. (2001). On the fragility of skilled performance: What governs choking under pressure? Journal of Experimental Psychology: General, 130, 701-725.

Beilock, S. L., Carr, T. H., Mac Mahon, C., \& Starkes, J. L. (2002). When paying attention becomes counterproductive: Impact of divided versus skill-focused attention on novice and experienced performance of sensorimotor skills. Journal of Experimental Psychology: Applied, 8, 6-16.

Bell, J. J., \& Hardy, J. (2009). Effects of attentional focus on skilled performance in golf. Journal of Applied Sport Psychology, 21, 163-177.

Boutcher, S. H. (2008). Attentional processes and sport performance. In T.S. Horn (Ed.), Advances in sport psychology (pp. 325-338). Champaign, IL: Human Kinetics. 
Caliari, P. (2008). Enhancing forehand acquisition in table tennis: The role of mental practice. Journal of Applied Sport Psychology, 20, 88-96.

Calmels, C., Berthoumieux, C., \& Arripe-Longueville, F. (d') (2004). Effects of an imagery training program on selective attention of national softball players. The Sport Psychologist, 18, 272-296.

Cohen, J. (1960). A coefficient of agreement for nominal scales. Educational and Psychological Measurement, 20, 37-46.

Crews, D. J., \& Boutcher, S. H. (1986). Effects of structured preshot behaviors on beginning golf performance. Perceptual and Motor Skills, 62, 291-294.

Cumming, J., Clark, S. E., Ste-Marie, D. M., McCullagh, P., \& Hall, C. (2005). The Functions of Observational Learning Questionnaire (FOLQ). Psychology of Sport and Exercise, 6, 517-537.

Eysenck, M. W. \& Calvo, M. G. (1992). Anxiety and performance: The processing efficiency theory. Cognition and Emotion, 6, 409-434.

Fleiss, J. L. (1981). Statistical methods for rates and proportions. New York: Wiley.

Ford, P., Hodges, N. J., \& Williams, A. M. (2005). Online attentional-focus manipulations in a soccerdribbling task: Implications for the proceduralization of motor skills. Journal of Motor Behavior, 37, 386-394.

Fournier, J. F., Deremaux, S., \& Bernier, M. (2008). Content, characteristics and function of mental images. Psychology of Sport and Exercise, 9, 734-748.

Gucciardi, D. F., \& Dimmock, J. A. (2008). Choking under pressure in sensorimotor skills: Conscious processing or depleted attentional resources. Psychology of Sport and Exercise, 9, 45-59.

Hanton, S., Mellalieu, S. D., \& Hall, R. (2004). Self-confidence and anxiety interpretation: A qualitative investigation. Psychology of Sport and Exercise, 5, 477495.

Hardy, L., Mullen, R., \& Jones, G. (1996). Knowledge and conscious control of motor actions under stress. British Journal of Psychology, 87, 621-636.

Hars, M., \& Calmels, C. (2007). Observation of elite gymnastic performance: Processes and perceived functions of observation. Psychology of Sport and Exercise, 8, 337354.

Hutchinson, J. C., \& Tenenbaum, G. (2007). Attention focus during physical effort: The mediating role of task intensity. Psychology of Sport and Exercise, 8, 233-245.

Jeannerod, M. (2001). Neural simulation of action: A unifying mechanism for motor cognition. NeuroImage, 14, S103-S109.

Jonides, J. (1981). Voluntary versus automatic control over the mind's eye's movement. In J. B. Long \& A. D. Baddeley (Eds.), Attention and performance IX (pp. 187-203). Hillsdale, NJ: Erlbaum.

Lincoln, Y. S., \& Guba, E. G. (1985). Naturalistic inquiry. Newbury Park, CA: Sage.

Loze, G. M., Holmes, P., Collins, D., \& Bellamy, M. (1998, June). An electroencephalographic study of elite UIT air-pistol shooters. Paper presented at the British Association of Sport and Exercise Sciences Annual Conference, Worcester, UK. 
Magill, R. A. (2003). Motor learning and control: Concepts and applications (7th ed.). New York: McGraw-Hill.

Masters, R. S. W. (1992). Knowledge, knerves and know-how: The role of explicit versus implicit knowledge in the breakdown of a complex motor skill under pressure. British Journal of Psychology, 83, 343-358.

McNevin, N. H., Shea, C. H., \&Wulf, G. (2003). Increasing the distance of an external focus of attention enhances learning. Psychological Research, 67, 22-29.

Morgan, W. P., \& Pollack, M. L. (1977). Psychological characterization of the elite distance runner. Annals of the New York Academy of Sciences, 44, 80-87.

Murphy, S., Nordin, S. M., \& Cumming, J. (2008). Imagery in sport, exercise and dance. In T. Horn (Ed.), Advances in sport and exercise psychology (3rd ed; pp. 297-324). Champagne, IL: Human Kinetics.

Nideffer, R. M., \& Sagal, M. S. (1998). Concentration and attention control training. In Williams, J.M. (Ed.), Applied sport psychology: Personal growth to peak performance, 3rd ed., pp. 296-315, Palo Alto, CA: Mayfield.

Nordin, S. M., \& Cumming, J. (2005). Professional dancers describe their imagery: Where, when, what, why, and how. The Sport Psychologist, 19, 395-416.

Oudejans, R. R., Kuijpers, W., Kooijman, C. C., \& Bakker, F. C. (2011). Thoughts and attention of athletes under pressure: Skill-focus or performance worries? Anxiety, Stress \& Coping, 24, 59-73.

Patton, M. Q. (2002). Qualitative evaluation and research methods (3rd ed). Thousand Oaks, CA: Sage.

Perkins-Ceccato, N., Passmore, S. R.,\&Lee, T. D. (2003). Effects of focus of attention depend on golfers' skill. Journal of Sport Sciences, 21, 593-600.

Posner, M. I. (1980). Orienting of attention. Quarterly Journal of Experimental Psychology, 32, 3-25.

Posner, M. I., \& Boies, S. J. (1971). Components of attention. Psychological Review, 78, 391-408.

Schomer, H. H. (1986). Mental strategies and the perception of effort of marathon runners. International Journal of Sport Psychology, 17, 41-59.

Singer, R. N. (2002). Preperformance state, routines, and automaticity: What does it take to realize expertise in self-paced events? Journal of Sport and Exercise Psychology, $24,359-375$.

Sparkes, A., \& Smith, B. (2009). Judging the quality of qualitative inquiry: Criteriology and relativism in action, Psychology of Sport \& Exercise, 10, 491-497.

Tenenbaum, G., Lloyd, M., Pretty, G., \& Hanin, Y. (2002). Congruence of actual and retrospective reports of pre-competition emotions in equestrians. Journal of Sport and Exercise Psychology, 24, 271-288.

Trudel, P., Haughian, L., \& Gilbert, W. (1996). L'utilisation de la technique du rappel stimulé pour mieux comprendre le processus d'intervention de l'entraîlneur en sport [Use of the stimulated recall technique to better understand the intervention process in sport coaching]. Revue des Sciences de l'Education, 22, 503-522. 
von Cranach,M., \& Harré, R. (1982). The analysis of action: Recent theoretical and empirical advances. Cambridge: Cambridge University Press.

Watkins, M. W. (2002). MacKappa [computer software]. University Park, PA: The Pennsylvania State University.

Williams, A. M., \& Ericsson, K. A. (2005). Perceptual-cognitive expertise in sport: Some considerations when applying the expert performance approach. Human Movement Science, 24, 283-307.

Wilson, T. D. (1994). The proper protocol: Validity and completeness of verbal reports. Psychological Science, 5(5), 249-252.

Wine, J. (1971). Test anxiety and direction of attention. Psychological Bulletin, 76, 92104.

Wulf, G. (2007). Attention and motor skill learning. Champaign, IL: Human Kinetics.

Wulf, G., \& Prinz, W. (2001). Directing attention to movement effects enhances learning: A review. Psychonomic Bulletin \& Review, 8, 648-660.

Wulf, G., \& Shea, C. H. (2002). Principles derived from the study of simple skills do not generalize to complex skills learning. Psychonomic Bulletin and Review, 9, 185-211.

Wulf, G., \& Su, J. (2007). An external focus of attention enhances golf shot accuracy in beginners and experts. Research Quarterly for Exercise and Sport, 78, 384-389.

The authorswish to thank the elite golfers who participated in this study. The study was undertaken with research grants from the French Ministry of Sport and from the French Federation of Golf. Additional thanks to Laura Killian, David Mann and Rémy Dinh-Phung for their comments on the manuscript. 


\section{Table 1}

\section{Categorization of the MUs}

\begin{tabular}{|c|c|c|c|}
\hline \multirow[b]{2}{*}{$\begin{array}{l}\text { Content } \\
(688 \mathrm{MUs}, 100.00 \%) \\
k=.89, z=14.94^{*} \\
\end{array}$} & \multicolumn{3}{|c|}{ Characteristics } \\
\hline & $\begin{array}{c}\text { Sense } \\
(614 \mathrm{MUs}, 100.00 \%) \\
k=.75, z=11.20^{*}\end{array}$ & $\begin{array}{c}\text { Reality } \\
(688 \mathrm{MUs}, 100.00 \%) \\
k=.75, z=6.48^{*}\end{array}$ & $\begin{array}{c}\text { Deliberateness } \\
(688 \mathrm{MUs}, 100.00 \%) \\
k=.66, z=4.59^{*}\end{array}$ \\
\hline $\begin{array}{l}\text { Process ( } 294 \mathrm{MUs}, 42.73 \% \text { ) } \\
\text { Result ( } 272 \mathrm{MUs}, 39.53 \%) \\
\text { Effects on the ball }(268 \mathrm{MUs}, 38.95 \% \text { ) } \\
\text { Score (4MUs, } 0.58 \% \text { ) } \\
\text { Psychological state ( } 74 \mathrm{MUs}, 10.75 \% \text { ) } \\
\text { Environment ( } 48 \mathrm{MUs}, 6.98 \% \text { ) } \\
\text { Related to the task (44 MUs, } 6.40 \% \text { ) } \\
\text { Not related to the task (4 MUs, } 0.58 \% \text { ) }\end{array}$ & $\begin{array}{l}\text { Visuai (405 MUs, } \\
65.96 \%) \\
\text { Kinesthetic ( } 204 \\
\text { MUs, } 33.22 \% \text { ) } \\
\text { Auditory (5 MUs, } \\
0.82 \% \text { ) }\end{array}$ & $\begin{array}{l}\text { Real }(550 \mathrm{MUs}, \\
79.94 \%) \\
\text { Imagined (138 MUs, } \\
20.06 \%)\end{array}$ & $\begin{array}{l}\text { Deliberate (606 MUs, } \\
\quad 88.08 \% \text { ) } \\
\text { Spontaneous ( } 82 \text { MUs, } \\
\quad 11.92 \% \text { ) }\end{array}$ \\
\hline
\end{tabular}

Note. " $p<.0001$

Table 2

Percentages of each category of content and characteristics in training and competition

\begin{tabular}{lrr}
\hline & Training & Competition \\
\hline Content & $100.00 \%$ & $100.00 \%$ \\
Process & $60.33 \%$ & $29.20 \%$ \\
Result & $31.67 \%$ & $45.73 \%$ \\
Psichological state & $5.67 \%$ & $14.47 \%$ \\
Enwironment & $2.33 \%$ & $10.60 \%$ \\
Sense & $100.00 \%$ & $100.00 \%$ \\
Visual & $57.60 \%$ & $73.03 \%$ \\
Kinesthetic & $40.99 \%$ & $26.67 \%$ \\
Anditory & $1.41 \%$ & $0.30 \%$ \\
Reality & $100.00 \%$ & $100.00 \%$ \\
Real & $81.00 \%$ & $79.12 \%$ \\
Inagined & $19.00 \%$ & $20.88 \%$ \\
Deliberateness & $100.00 \%$ & $100.00 \%$ \\
Deliberate & $90.00 \%$ & $86.60 \%$ \\
Spontaneous & $10.00 \%$ & $13.40 \%$ \\
\hline
\end{tabular}

